\title{
Subcutaneous Immunoglobulin in Chronic Inflammatory Demyelinating Polyneuropathy - Neurologist, Nursing and Patient Perspectives
}

\author{
Hans Katzberg, ${ }^{1}$ Richard A Lewis, ${ }^{2}$ Thomas Harbo, ${ }^{3}$ Jeffrey A Allen, ${ }^{4}$ Melody Bullock, ${ }^{5}$ Dorothea Grosse-Kreul, ${ }^{6}$ \\ James Babington Smith7 and Vera Bril1,8 \\ 1. Ellen and Martin Prosserman Centre for Neuromuscular Diseases, Division of Neurology, Department of Medicine, University Health \\ Network, University of Toronto, Toronto, Canada; 2. Department of Neurology, Cedars-Sinai Medical Center, Los Angeles, CA, USA; \\ 3. Department of Neurology, Johns Hopkins Medicine, Baltimore, MD, USA; 4. Department of Neurology, University of Minnesota, \\ Minneapolis, MN, USA; 5. CSL Behring, King of Prussia, PA, USA; 6. Department of Allergy and Clinical Immunology, Division of Asthma, \\ Allergy and Lung Biology, King's College London, King's Health Partners, King's College Hospital NHS Foundation Trust, School of Medicine, \\ London, UK; 7. Patient Representative on GAIN Medical Advisory Board, Sleaford, Lincolnshire, UK; 8. Institute for Research and Medical \\ Consultations, Imam Abdulrahman Bin Faisal University, Dammam, Saudi Arabia
}

in mmunoglobulin (Ig) therapy is a common treatment for chronic inflammatory demyelinating polyneuropathy (CIDP). Intravenous immunoglobulins (IVIg) have been shown to be efficacious in CIDP; however, many patients experience fluctuations in functionality throughout the treatment cycle, systemic adverse events (AES) and inconvenience due to hospital-based treatment. An alternative to IVIg is subcutaneous immunoglobulin (SCIg). Several studies have demonstrated the efficacy of SCIg in CIDP, with the recent PATH study leading to the approval of the $20 \%$ SCIg IgPro20 (Hizentra ${ }^{\circledR}$ ) by agencies including the US Food and Drug Administration, Health Canada and the European Medicine Agency. SCIg administration differs from IVIg and therefore requires patients and nurses to be adequately trained in self-administration techniques and treatment monitoring. SCIg can be administered via a manual push method or using a pump. Training patients to self-administer is straightforward; however, current efficacy monitoring assessments could be improved to better suit home-based therapy. Preference for SCIg is often due to increased autonomy, reduced systemic AEs and reduced fluctuations in functionality; but ultimately, the preference for SCIg or IVIg will often depend upon a patient's individual personality and lifestyle.

\section{Keywords}

Chronic inflammatory demyelinating polyneuropathy, patient perspective, subcutaneous immunoglobulin, intravenous immunoglobulins

Disclosure: Hans Katzberg has acted as a consultant for Shire, CSL Behring, Flexpharma, Grifols, Octapharma, Biogen, Pfizer, Sanofi and Genzyme; is a member of the Muscle Study Group and is on the medical advisory board of the Myasthenia Gravis Foundation of America and the GBS/CIDP Foundation of Canada. Richard A Lewis is the chair of the Inflammatory Neuropathy Consortium (INC), a standing committee of the Peripheral Nerve Society; is on the Board of Directors of the Peripheral Nerve Society and a Medical Advisory Board member of the CIDP/ GBS Foundation International; is a member of the Myasthenia Gravis Foundation of America, and of the Myasthenia Gravis Foundation of California; is a paid consultant for CSL Behring, Novartis, Pharnext, Axelacare, Biotest and Nufactor. Thomas Harbo is a board member of the Inflammatory Neuropathy Consortium (INC), a standing committee of the Peripheral Nerve Society, and is a paid consultant for CSL Behring. Jeffrey Allen is a board member of the Inflammatory Neuropathy Consortium (INC), a standing committee of the Peripheral Nerve Society and is a paid consultant for CSL Behring, Biotest and ArgenX. Melody Bullock is an employee of CSL Behring. Dorothea GrosseKreul has received lecture fees, advisory board and travel expense support from CSL Behring. Shire, Grifols,

Biotest and BPL. James Babington Smith has received fees and travel expenses from CSL Behring. Vera Bril is a consultant to CSL Behring, Grifols, Union Chimique Belge, Takeda (Shire), Bionevia, Alnylam, Pfizer and ArgenX; serves on international scientific advisory boards for the Myasthenia Gravis Foundation of America and GuillainBarré Syndrome/Chronic Inflammatory Demyelinating Polyneuropathy (CIDP/GBS) Foundation International; and has received research support from CSL Behring, Grifols, Bionevia, Union Chimique Belge, Biogen and ArgenX.

Review Process: Double-blind peer review.

Acknowledgements: Editorial assistance was provided by Joanna Musgrove of Meridian HealthComms, funded by CSL Behring.

Compliance with Ethics: All procedures were followed in accordance with the responsible committee on human experimentation and with the Helsinki Declaration of 1975 and subsequent revisions, and informed consent was received from the patient involved in this case study.

Authorship: All named authors meet the International Committee of Medical Journal Editors (ICMJE) criteria for authorship of this manuscript, take responsibility for the integrity of the work as a whole, and have given final approval for the version to be published.

Received: 7 March 2019

Accepted: 20 June 2019

Citation: European Neurological Review. 2019;14(1):44-9

Corresponding Author: Hans Katzberg, Toronto General Hospital/UHN Room 306,

200 Elizabeth Street, Toronto, Ontario, Canada, M5G 2C4. E: hans.katzberg@utoronto.ca
Chronic inflammatory demyelinating polyneuropathy (CIDP) is a peripheral neuropathy that can affect motor and/or sensory nerves, and lead to both loss of strength and altered sensation. ${ }^{1}$ Common firstline treatments for CIDP are immunoglobulins (Igs), corticosteroids and plasma exchange. ${ }^{2}$ Intravenous immunoglobulins (IVIgs) have been shown to be efficacious in maintaining or improving strength and disability in a number of CIDP studies. ${ }^{3,4}$ Limitations of IVIg include relatively frequent systemic adverse events (AEs), the most common of which is headache. ${ }^{3,4}$ In addition, although some companies offer home-based IVIg infusions, in most countries the majority of IVIg infusions are given within hospitals or infusion centres.3.5,6 Furthermore, while IVIg is an established treatment for CIDP, it may be associated with fluctuations in functionality throughout the treatment cycle.

An alternative to $\mathrm{IVIg}$ is subcutaneous immunoglobulin (SClg). SClg is preferred by many patients because it allows for convenient home-based self-administration, reducing the need for hospital or infusion centrebased treatments. Subcutaneously infused Igs slowly enter the vascular space, with 
Table 1: Comparison of intravenous and subcutaneous immunoglobulin parameters

\begin{tabular}{|c|c|c|}
\hline Parameter & IVIg & SClg \\
\hline Concentration* & • $10 \%$ & • $20 \%$ \\
\hline Volume injected & - $80 \mathrm{~kg}$ patient, $1 \mathrm{~g} / \mathrm{kg}=80 \mathrm{~g}(800 \mathrm{~mL})$ & - $80 \mathrm{~kg}$ patient, $0.2 \mathrm{~g} / \mathrm{kg}=16 \mathrm{~g}(80 \mathrm{~mL})$ \\
\hline Infusion frequency & - Typically every 3 weeks & - Given weekly, twice weekly or variably ${ }^{\dagger}$ \\
\hline Infusing personnel & $\begin{array}{l}\text { - Administration by a trained health care professional is } \\
\text { usually required }\end{array}$ & - Can be self-administered \\
\hline Site of care & - Infusion centre/clinic or given during home nursing visits & - Home \\
\hline Duration of infusion & - Infusion can last 4-6 hours” & $\begin{array}{l}\text { - Infusion lasts approximately } 1 \text { hour }{ }^{\sharp} \\
\text { - Manual push SClg infusions can last approximately } \\
\text { 5-6 minutes (infusing at } 1 \mathrm{~mL} / \text { minute) })^{\#}\end{array}$ \\
\hline Infusion sites & - Can be infused into a single site through a dedicated port & $\begin{array}{l}\text { - Can infuse into multiple sites at once } \\
\text { (range 2-8 sites) }\end{array}$ \\
\hline Benefits & $\begin{array}{l}\text { - Infusions occur less frequently and patients can attend an } \\
\text { infusion clinic for their medication } \\
\text { - Some patients may benefit from additional medical } \\
\text { monitoring }\end{array}$ & $\begin{array}{l}\text { - Can be self-administered and provides patients } \\
\text { increased autonomy, allowing patients to continue } \\
\text { with their daily lives. Infusions take less time than } \\
\text { those with IVIg. Allows patients with venous access } \\
\text { issue to continue Ig therapy }\end{array}$ \\
\hline Adverse events & $\begin{array}{l}\text { - More chance of systemic reactions including headaches and } \\
\text { nausea }\end{array}$ & - More chance of local reactions \\
\hline
\end{tabular}

* Other concentrations are available for IVIg and SCIg products; ${ }^{\dagger}$ Infusion frequency can be tailored to the needs of the individual patients; \#Time for infusion, additional time is required to prepare infusion; $I g=$ immunoglobulin; IVIg = intravenous immunoglobulin; $S C l g=$ subcutaneous immunoglobulin.

maximum serum immunoglobulin $\mathrm{G}(\mathrm{IgG})$ levels reached approximately 2 days after infusion, ${ }^{8}$ compared with IVIg which enters the vascular space within several hours; 9 the slower diffusion of SClg likely leads to the observed fewer systemic AEs such as headache. ${ }^{10-12}$ Additional comparisons between IVIg and SCIg are shown in Table $1 .{ }^{13}$ Of note, due to dedicated manufacturing steps designed to remove or inactivate viruses and other pathogens, both IVIg and SCIg present a very low risk of disease transmission. ${ }^{14-16}$ Indeed, to date there have been no cases of transmission of viral infection or Creutzfeldt-Jakob disease associated with the use of currently available Ig products. In addition, no cases of progressive multifocal leukoencephalopathy (JC virus reactivation) have been seen with IVIg or SCIg. Here we detail a case study of a patient switching from IVIg to SCIg and review the relevant literature from the neurologist, nurse and patient perspective, including details on the recent large-scale Polyneuropathy And Treatment with Hizentra (PATH) study. ${ }^{10}$

\section{Patient case study}

The patient became ill with acute Guillain-Barré syndrome in 1989. After achieving a partial recovery, he was left with significant disability; although his core strength slowly improved over the next few years. In 2005, he experienced symptoms including weakness in the arms and legs, 'pins and needles', neuropathic pain and difficulty walking, and was referred to a neurologist. Raised cerebrospinal fluid protein measurements and overall progression of disability over $>2$ months, combined with meeting the European Federation of Neurological Societies/Peripheral Nerve Society (EFNS/PNS) criteria for CIDP, resulted in a diagnosis of CIDP. This was further supported by electromyography results consistent with CIDP the following year. The patient was treated with IVIg (2005-2011), SClg administered via pump (2012-2014) and manual push SClg (2014-present). A summary of the patient's experience with all three methods is shown in Box 1.

After IVIg treatment the patient felt considerably stronger, walking ability and manual dexterity improved and pain levels were reduced; although wear-off of treatment effects was experienced between infusions. It was common to experience systemic AEs at the end of the first day of infusions including headache, stomach ache and nausea; however, these did not persist.

Whilst the patient was initially treated with IVIg, when his hospital began offering $\mathrm{SClg}$, the patient opted for this administration method. With SClg administered via pump, the patient and his caregiver were trained in the preparation and administration of SClg. This patient was unable to draw up the product into the syringe; however, once the product was drawn up into the syringe, the rest of the administration process could be undertaken by the patient. The patient did not experience any noticeable AEs with this treatment, with only transient swelling at the site of infusion. With the manual push SClg method, the patient was able to perform all steps of the infusion without assistance. Stabilisation of symptoms was similar with both Sclg administration methods. Whilst the patient initially experienced some wear-off over time, efficacy was stabilised by increasing the SClg dose, leading to efficacy similar to peak IVIg. On SClg, the patient experienced reduced wear-off effects compared with IVIg.

\section{Evidence of subcutaneous immunoglobulin efficacy in neuropathies - a neurologist's perspective from the literature}

Treatment efficacy is a major consideration for neurologists when choosing a suitable treatment for patients with CIDP. Several studies have demonstrated the efficacy of SClg in neuropathies. Markvardsen et al. recently reviewed 20 publications on SCIg in CIDP or multifocal motor neuropathy (MMN) and concluded that muscle strength, function and ability can be maintained with SCIg treatment in both disorders. SCIg was also associated with fewer systemic AEs compared with IVIg. ${ }^{17}$ A metaanalysis conducted by Racosta et al. analysed data from eight studies, with a total of 138 patients (50 with MMN and 88 with CIDP). ${ }^{18}$ No significant difference was observed in muscle strength outcomes between SCIg and IVIg, but an improved AE profile was observed in patients with MMN and CIDP receiving SCIg compared with IVIg. ${ }^{18}$ Long-term response to SCIg has recently been reported by Cirillo et al., who demonstrated improvement 


\section{Box 1: Patient case study}

\section{Diagnosis}

- The patient became ill with acute Guillain-Barré syndrome in 1989.

- After achieving a partial recovery, he was left with significant disability, although his core strength slowly improved over the next few years.

- In 2005, he experienced symptoms including weakness in the arms and legs, 'pins and needles', neuropathic pain and difficulty walking, and was referred to a neurologist, who, with the support of cerebrospinal fluid protein measurement and electromyography tests, confirmed a diagnosis of chronic inflammatory demyelinating polyneuropathy (CIPD).

\section{Treatment}

Intravenous immunoglobulin (IVIg; 2005-2011)

- IVIg treatment comprised $80 \mathrm{~g}$ of a $10 \%$ immunoglobulin product every 28 days, infused over 2 days at $~ 90 \mathrm{~mL} /$ hour.

- Approximately 8-9 days after treatment, the patient felt considerably stronger, walking ability and manual dexterity improved and pain levels were reduced. This lasted for approximately 12 days before functionality would decline.

- It was common to experience headache, nausea, or stomach pain at the end of the first day of infusions; however, these did not persist into the second day of treatment.

- The treatments were hard to fit in around daily life.

o Product availability and venous access were also issues with IVIg.

\section{Subcutaneous immunoglobulin (SCIg; via pump 2012-2014 and manual push 2014-present)}

- The patient was switched to SCIg with a Crono pump (Cané, Rivoli, Italy).

- SClg infusions were twice-weekly, infusing $180 \mathrm{~mL}$ per week, over two sessions taking 2 hours per session.

- The patient and his caregiver were trained in the preparation and administration of SClg. The caregiver had to draw up the product into syringes; the rest of the administration process could be undertaken by the patient.

- SCIg administration via pump resulted in stabilisation of functionality once the ideal dose had been determined.

- The patient did not experience any noticeable side effects with this treatment, with only transient swelling at the site of the infusion.

- In 2015, the patient changed to manual push SCIg infusions. Infusions of $28 \mathrm{~g} /$ week were undertaken, usually infusing every other day.

- The patient was trained on administration by a specialist nurse, and was able to perform all steps of the infusion without assistance.

- Stabilisation of symptoms was similar with both SCIg administration methods.

- In terms of adverse events, the subcutaneous deposit was more noticeable with the manual push method.

of global strength, sensory deficits and overall disability in patients with CIDP treated with SCIg for 12 or 24 months. ${ }^{19}$

\section{The PATH study}

The recent PATH study (ClinicalTrials.gov identifier: NCT01545076) confirmed the efficacy of SClg as maintenance therapy in CIDP in a large-scale, placebo-controlled, randomised setting. ${ }^{10}$ This led to the approval of the $20 \%$ SCIg IgPro20 (Hizentra ${ }^{\circledR}$, CSL Behring, King of Prussia, PA, USA) for maintenance treatment of CIDP by authorities in several countries, including by the US Food and Drugs Administration, Health Canada and the European Medicines Agency.14,15,20

Prior to randomisation to either weekly $0.2 \mathrm{~g} / \mathrm{kg}$ or $0.4 \mathrm{~g} / \mathrm{kg} \mathrm{SClg}$ (IgPro20) or placebo for 24 weeks, patients underwent an IgG dependency period and IVIg restabilisation period to confirm their need for, and response to, IgG therapy. The primary endpoint of rate of relapse ( $\geq 1$-point deterioration in adjusted Inflammatory Neuropathy Cause and Treatment [INCAT] score) or withdrawal was 33\% for $0.4 \mathrm{~g} / \mathrm{kg}$ IgPro20 and $39 \%$ for $0.2 \mathrm{~g} / \mathrm{kg}$ IgPro20, both significantly lower ( $p=0.001, p=0.007$, respectively) than the $63 \%$ for placebo. Efficacy measures such as INCAT, grip strength, Medical Research Council sum scores and Inflammatory Rasch-built Overall Disability Score (I-RODS) remained more stable with IgPro20 compared with placebo. ${ }^{10}$

The most common AEs were local reactions at the infusion site and these were mostly mild in severity and decreased over time. The rate of headache was low in the PATH study (7\% of patients treated with SCIg experienced headache compared with $3.5 \%$ of placebo-treated patients.

\section{Assessing efficacy with home-based subcutaneous immunoglobulin}

When assessing the efficacy of Sclg, ensuring patients adequately monitor their response to treatment with a home-based therapy can be a challenge. Measuring serum IgG levels as a correlate with response does not appear to be beneficial from the PATH study results; however, training in the use of Vigorimeters, or other grip strength devices such as a Jamar handgrip dynamometer, and completion of patient-reported outcome measures such as the I-RODS questionnaire at home can assist in self-monitoring. ${ }^{3,21-23}$ It is also important to reassure patients that small fluctuations in functionality are common and do not necessarily indicate relapse. Development of new outcome measures that are more suited to home-based, patient-led assessment may be useful for the ongoing use of SCIg in CIDP.

\section{Practicalities of subcutaneous immunoglobulin - a nurse's perspective}

sclg can be administered via the manual push method or using a pump. Different pumps have advantages and disadvantages including size/portability, infusion rate and volume limits, or added features (e.g., blockage alarms). Two of the most common pumps available are the Crono (Cané, Rivoli, Italy) and the Freedom (RMS Medical Products, Chester, NY, USA) pumps (Figure 1). The battery-powered Crono pump is small and portable (pocket-sized); the infusion speed can be modified, an infusion time is set and it will infuse in that time. The Crono pump requires some dexterity and sensitivity to change the settings, which may be a challenge for some patients. The Freedom pumps are larger and are based on a wind-up mechanism rather than battery power. 


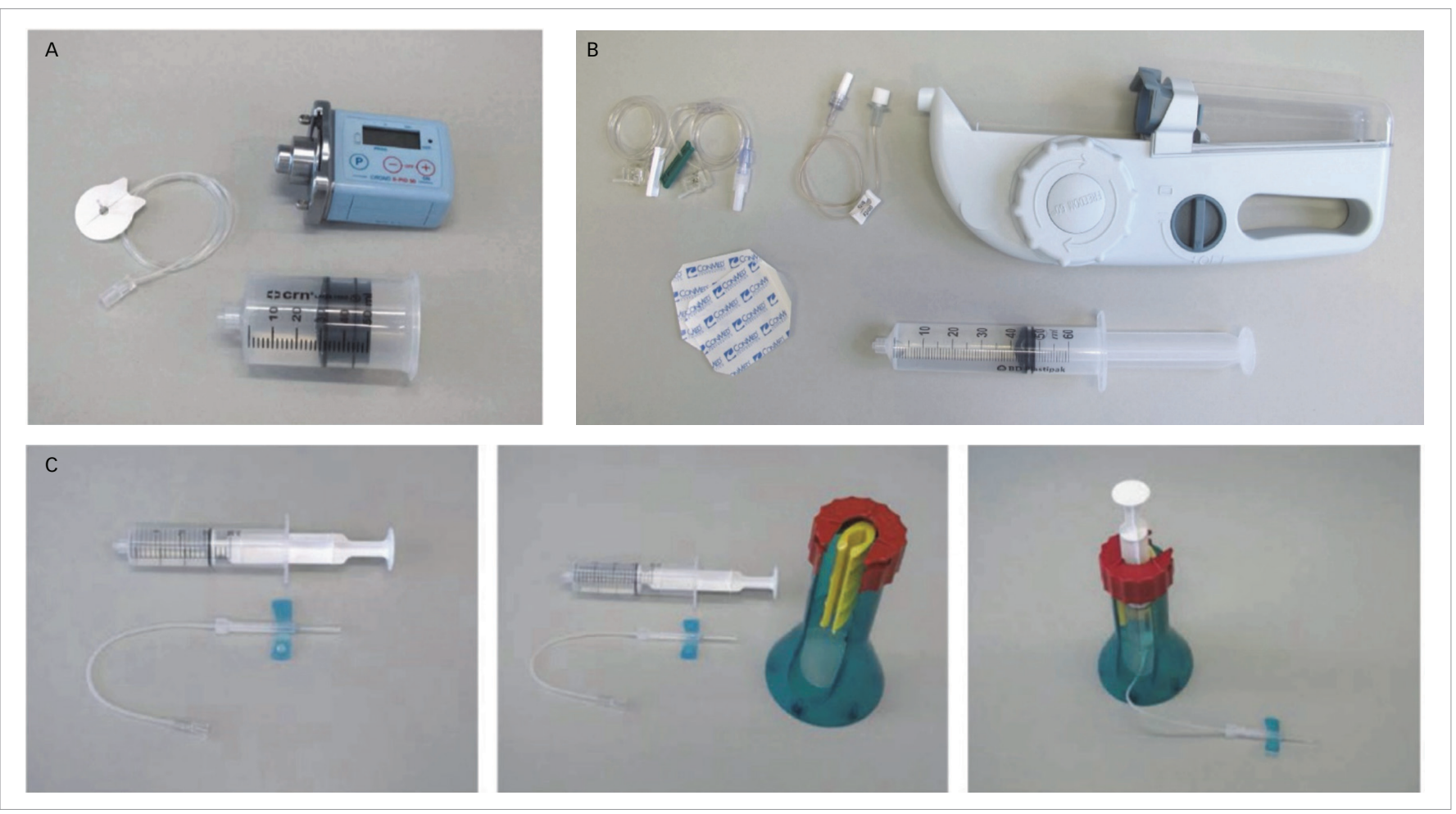

Crono pump (Cané, Rivoli, Italy); Freedom pump (RMS Medical Products, Chester, NY, USA); SteadyJect (CSL Behring, King of Prussia, PA, USA).

The speed of infusion is regulated by the lumen (and therefore the pressure) of the rate tubing; infusions can start with a lower lumen/ pressure to get patients used to the infusion and then build up if well tolerated. Other pumps are available, such as the SCIg 60 infuser (EMED, El Dorado Hills, CA, USA), and it is important to discuss the range of administration options available to patients to ascertain the best fit for each patient's lifestyle and personality.

As switching from IVIg to SCIg can mean a change from hospital- or clinic-based administration to home-based self-administration, ensuring that patients are infusing correctly can be a challenge. For patients with dexterity issues, syringe holders can be used to make infusions easier to manage; the availability of pre-filled syringes in certain regions has been shown to be of great benefit to patients with dexterity issues to reduce errors in preparation of infusion equipment. ${ }^{24}$ One of the major advantages when switching from IVIg to SClg is the avoidance of IV infusions; SC infusions require minimal training, can be completed by patients in their own homes and ensure that patients with IV access issues can benefit from Ig therapy. ${ }^{25,26}$ In the PATH study, most patients found the subcutaneous administration technique easy to use and all patients or caregivers learnt to effectively administer IgPro20 or placebo by themselves after four or fewer training sessions. ${ }^{27}$ Resources such as the International Nurses Group for Immunodeficiency guidelines on Ig administration and Train the Trainer video, ${ }^{28}$ as well as the Hizentra. com self-administration documents and videos ${ }^{29}$ can support training in Sclg use. Often a specialty pharmacy provider will train patients on using SClg in the home over 3-5 sessions and may assess patients via clinic follow-ups or phone assessments to monitor dose, volume, rate, tolerability, compliance and treatment barriers.

Suitable infusion sites for SClg are the abdomen, thigh, upper arm, and/or lateral hip, staying $\geq 2 \mathrm{~cm}$ ( $\geq 1 \mathrm{inch}$ ) away from any scar tissue. It is often recommended to rotate the site of infusion so it is not in the same location as the previous site; however, this is not always necessary and can be based on patient preference. If infusing at multiple sites at once, these sites should be $\geq 2$ inches $(5 \mathrm{~cm}$ ) apart. In the PATH study, the maximum infusion volume per site was $50 \mathrm{~mL}$ ( $\leq 20 \mathrm{~mL} / \mathrm{site}$ for the first infusion), and patients used a maximum of eight infusion sites in parallel, most, however, used between two and four needles. ${ }^{10,30}$ The maximum infusion rate per hour per site in the PATH study was $50 \mathrm{~mL}$ ( $\leq 20 \mathrm{~mL} /$ hour/site for the first infusion), SCIg infusions can therefore last less than an hour. SClg dosing can be divided and given at various intervals including daily to weekly or every other week with the same benefit as IVIg; however, some patients may require increases in their dosing compared with their previous IVIg dose. When discussing sites with patients, it is important to highlight that a site is usually considered a $2.5 \mathrm{~cm}^{2}$ area, approximately $1 \mathrm{~cm}$ around the site of injection, rather than an exact needle position. Transient local reactions can occur following Sclg infusion (Figure 2), these reactions usually subside within 24 hours. If local reactions such as redness, swelling or itching occur during infusion, the infusion can be paused while a heat pack is applied to help with absorption of SCIg and reducing the reaction (redness from the heat application may be present following this and will fade after a short time). Alternatively, changing ancillary supplies such as the tape, transparent dressing, or skin preparation supplies may be suggested. Additionally, further training to confirm technique and optimise needle length, infusion volume and flow rate and infusion site selection may be useful.

\section{Patient Perspectives}

From patient-reported outcomes in the literature, the benefits of SClg versus IVIg appear to include reduced systemic AEs, the option to split infusions over different sessions or multiple sites (flexible dosing) and reduction of wear-off effects (Table 1). ${ }^{13,31}$ In addition, compared with IVIg (5-6 hours per infusion), sclg allows for rapid infusion (approximately 1 hour per infusion), which can be tailored to each patient's tolerance. ${ }^{14,32,33}$ The preference of IVIg versus SCIg will often depend 
Figure 2: Example of infusion site during (A) and following subcutaneous immunoglobulin infusion (B-D)

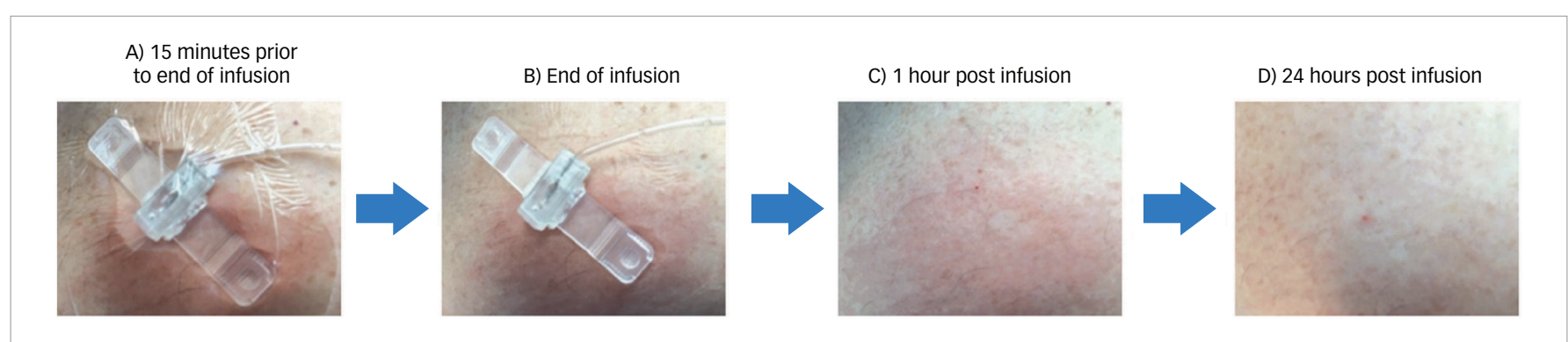

A mild injection site reaction can be observed following subcutaneous immunoglobulin (SClg) infusion. A moderate reaction can be seen at the end of SClg infusion period which dissipates to a mild reaction 1-hour post infusion. After 24 hours the infusion site shows minimal reaction. The above infusion took 35 minutes, infusing 40 mL SClg into the abdomen.

upon a patient's individual personality and lifestyle; some patients may prefer to have IV infusions in a healthcare setting and enjoy the social aspect of infusion centres, others may prefer the convenience and autonomy of SCIg. The patient in this case study experienced reduced wear-off effects when switching from IVIg to SClg, potentially due to more stable serum IgG levels with SCIg. SCIg provides more constant IgG levels compared with IVIg resulting in reduced wear-off effects at the end of treatment cycles. ${ }^{1,34-36}$ During the PRIMA (Privigen ${ }^{\circledast}$ Impact on Mobility and Autonomy) trial, peaks of 32.3 (SD 8.0) g/L and trough levels of 17.5 (3.1) g/L were seen with IVIg $1 \mathrm{~g} / \mathrm{kg}$ every 3 weeks, ${ }^{4}$ whilst in PATH, patients experienced serum IgG trough levels of $15.3(2.57) \mathrm{g} / \mathrm{L}$ at a dose of $0.2 \mathrm{~g} / \mathrm{kg}$ and of 20.8 (3.23) $\mathrm{g} / \mathrm{L}$ with $0.4 \mathrm{~g} / \mathrm{kg}$. ${ }^{14}$

It should be highlighted that although the patient in our case study had a positive experience with $\mathrm{SClg}$, this would not be the ideal setting for patients who prefer fewer infusions or the hospital/clinic setting. The use of implanted ports for IVIg administration can be associated with complications, so SCIg administration may be more appropriate for patients with venous access issues. In a study by Hadden et al. in eight subjects (four with MMN and four with CIDP) who switched from IVIg to SClg there was a strong preference for SClg over IVIg, with perceived benefits of SClg including travel convenience and ease of self-administration. ${ }^{37}$ In a study by cocito et al., switching to home-based SClg improved quality of life index scores; the biggest improvements were seen for 'treatment not interfering with work', 'no anxiety', 'autonomy', 'convenience' and 'painless' domains after 6 months, suggesting these aspects can impact preference. ${ }^{11}$ In the
PATH study, 53\% of patients preferred current SClg treatment over pre-study IVIg treatment, while approximately $18 \%$ preferred IVIg..$^{10}$ The difference in preference in the PATH study was largely due to patients reporting that SCIg offered them more independence and autonomy, while patients who preferred IVIg did so primarily due to a better perceived efficacy of IVIg. ${ }^{10}$

\section{Conclusions}

SClg is a suitable alternative to IVIg for the treatment of CIDP in terms of efficacy and tolerability with individual patient preference a key factor in the choice of use of IVIg or SCIg, as shown in the real-world case study presented here and the literature reviewed. In comparison with IVIg, SClg is associated with fewer systemic AEs; if local reactions are experienced, these tend to be mild and become less frequent over time. Switching from IVIg to SClg is simple, with different administration options to suit patient needs and limited patient training required from nurses. In spite of weakness, numbness and manual dexterity limitations in patients with neuromuscular diseases, most patients with MMN or CIDP are able to perform subcutaneous injections either themselves or with help from their caregiver, giving patients more independence than with hospital-based IVIg. Despite these advantages, some patients may not tolerate sclg or show adequate clinical response to Sclg; therefore, close monitoring when switching or starting a patient on SClg is recommended. Additional clinical research into the use of SClg in the management of patients with chronic inflammatory neuropathies and other neuromuscular conditions is recommended. $\square$
1. Mathey EK, Park SB, Hughes RA, et al. Chronic inflammatory demyelinating polyradiculoneuropathy: from pathology to demyelinating polyradiculoneuropathy: from pathology to
phenotype. J Neurol Neurosurg Psychiatry. 2015;86:973-85. phenotype. J Neurol Neurosurg Psychiatry. 2015;86:973-85
Van den Bergh PY, Hadden RD, Bouche P, et al. European

2. Van den Bergh PY, Hadden RD, Bouche P, et al. European
Federation of Neurological Societies/Peripheral Nerve Federation of Neurological Societies/Peripheral Nerve
Society guideline on management of chronic inflammatory demyelinating polyradiculoneuropathy: report of a joint task force of the European Federation of Neurological Societies and the Peripheral Nerve Society - first revision. Eur J Neurol. 2010;17:356-63.

3. Hughes RA, Donofrio P, Bril V, et al. Intravenous immune globulin ( $10 \%$ caprylate-chromatography purified) for the treatment of chronic inflammatory demyelinating polyradiculoneuropathy (ICE study): a randomised placebo-controlled trial. Lancet Neurol. 2008;7:136-44.

4. Léger JM, De Bleecker JL, Sommer C, et al. Efficacy and safety of Privigen ${ }^{\oplus}$ in patients with chronic inflammatory safety of Privigen in patients with chronic inflammatory
demyelinating polyneuropathy: results of a prospective demyelinating polyneuropathy: results of a prospective,
single-arm, open-label Phase III study (the PRIMA study). single-arm, open-label Phase III study

5. Hughes RA. Intravenous immunoglobulin for chronic inflammatory demyelinating polyradiculoneuropathy: the ICE trial. Expert Rev Neurother. 2009;9:789-95.

6. Hughes RA, Dalakas MC, Cornblath DR, et al. Clinical applications of intravenous immunoglobulins in neurology. Clin Exp Immunol. 2009;158(Suppl 1):34-42.

7. Pollard JD, Armati PJ. CIDP - the relevance of recent advances in Schwann cell/axonal neurobiology. J Peripher Nerv Syst. 2011; 16:15-23.

8. Jolles S, Orange JS, Gardulf A, et al. Current treatment options with immunoglobulin $\mathrm{G}$ for the individualization of care in patients with primary immunodeficiency disease Clin Exp Immunol. 2015;179:146-60.

9. Bonilla FA. Pharmacokinetics of immunoglobulin administered via intravenous or subcutaneous routes. Immunol Allergy Clin North Am. 2008;28:803-19.

10. van Schaik IN, Bril V, van Geloven N, et al. Subcutaneous immunoglobulin for maintenance treatment in chronic inflammatory demyelinating polyneuropathy (PATH): a randomised, double-blind, placebo-controlled, phase 3 trial. Lancet Neurol. 2018;17:35-46.

11. Cocito D, Serra G, Paolasso I, et al. Economic and quality of life evaluation of different modalities of immunoglobulin therapy in chronic dysimmune neuropathies. J Peripher Nerv syst. 2012;17:426-8.

12. Markvardsen $\mathrm{LH}$, Christiansen I, Andersen $\mathrm{H}$, Jakobsen J. Headache and nausea after treatment with high-dose subcutaneous versus intravenous immunoglobulin. Basic Clin Pharmacol Toxicol. 2015;117:409-12.

13. Younger M. IDF Guide for Nurses: Immunoglobulin Therapy for Primary Immunodeficiency Diseases. 2013. Available at: https://primaryimmune.org/wp-content/uploads/2015/11//DFGuide-for-Nurses-2013.pdf (accessed 23 April 2019).

14. CSL Behring. Hizentra Prescribing Information. 2018. Available at: http://labeling.cslbehring.com/PI/US/Hizentra/EN/ Hizentra-Prescribing-Information.pdf (accessed 8 August 2018).

15. CSL Behring. Hizentra Summary of Product Characteristics. 2018. Available at: www.ema.europa.eu/docs/ en_GB/document_library/EPAR_-_Product_Information/ human/002127/WC500107057. pdf (accessed 8 August 2018).
16. Stucki M, Boschetti N, Schafer W, et al. Investigations of prion and virus safety of a new liquid IVIG product. Biologicals. 2008;36:239-47

17. Markvardsen LH, Harbo T. Subcutaneous immunoglobulin treatment in CIDP and MMN. Efficacy, treatment satisfaction and costs. J Neurol Sci. 2017;378:19-25.

18. Racosta JM, Sposato LA, Kimpinski K. Subcutaneous versus intravenous immunoglobulin for chronic autoimmune neuropathies: a meta-analysis. Muscle Nerve. 2017;55:802-9.

19. Cirillo G, Todisco V, Tedeschi G. Long-term neurophysiological and clinical response in patients with chronic inflammatory demyelinating polyradiculoneuropathy treated with subcutaneous immunoglobulin. Clin Neurophysiol. 2018;129:967-73.

20. CSL Behring. Hizentra Product Monograph. 2018. Available at: http://labeling.cslbehring.ca/PM/CA/Hizentra/EN/HizentraProduct-Monograph.pdf (accessed 17 January 2019).

21. Vanhoutte EK, Latov N, Deng C, et al. Vigorimeter grip strength in CIDP: a responsive tool that rapidly measures the effect of IVIG--the ICE study. Eur J Neurol. 2013;20:748-55.

22. van Nes SI, Vanhoutte EK, van Doorn PA, et al. Rasch-built Overall Disability Scale (R-ODS) for immune-mediated peripheral neuropathies. Neurology. 2011;76:337-45.

23. Hughes R, Bensa S, Willison $H$, et al. Randomized controlled trial of intravenous immunoglobulin versus oral prednisolone in chronic inflammatory demyelinating polyradiculoneuropathy. Ann Neurol. 2001;50:195-201.

24. Kafal AR, Vinh DC, Langelier MJ. Pre-filled syringes for immunoglobulin $\mathrm{G}(\mathrm{IgG})$ replacement therapy: clinical 
experience from other disease settings. Expert Opin Drug Deliv 2018;15:1199-209

25. Berger M. Subcutaneous administration of IgG. Immunol Allergy Clin North Am. 2008;28:779-802

26. Cocito D, Romagnolo A, Peci E, et al. Subcutaneous vs. intravenous immunoglobulin in CIDP: pharmacokinetic and clinical response. J Peripher Nerv Syst. 2016;21:114-6.

27. van Schaik IN, Bril V, van Geloven N, et al. Practical application of subcutaneous immunoglobulin for maintenance treatment in CIDP: the PATH study. Presented at the Peripheral Nerve Society Annual Meeting, 22-25 July 2018, Baltimore, MD, USA.

28. International Nursing Group for Immunodeficiences. Resources. Available at: https://ingid.org/resources/ (accessed 28 June 2019).

29. CSL Behring. Self-administering Hizentra ${ }^{\star}$. Available at: www.hizentra.com/self-administering (accessed 28 June 2019). 30. Berger M, Harbo T, Cornblath DR, Mielke O. IgPro20, the Polyneuropathy and Treatment with Hizentra ${ }^{\circledR}$ study (PATH), Polyneuropathy and reatment with Hizentra study (PATH), polyradiculoneuropathy with subcutaneous IgG. immunotherapy. 2018;10:919-33.

31. Markvardsen LH, Harbo T, Sindrup SH, et al. Subcutaneous Markvardsen $\mathrm{LH}$, Harbo T, Sindrup SH, et al. Subcutaneo
immunoglobulin preserves muscle strength in chronic inflammatory demyelinating polyneuropathy. Eur I Neurol. 2014;21:1465-70

32. Wasserman RL, Melamed I, Nelson RP Jr., et al. Pharmacokinetics of subcutaneous IgPro20 in patients with primary immunodeficiency. Clin Pharmacokinet. 2011;50:405-14.

33. CSL Behring. Privigen Prescribing Information. 2013. Available at www.privigen.com/prescribing-information (accessed 9 July 2019).

34. Hagan JB, Fasano MB, Spector S, et al. Efficacy and safety of a new $20 \%$ immunoglobulin preparation for subcutaneous administration, gPro20, in patients with primary

35. Berger M. Subcutaneous immunoglobulin replacement in primary immunodeficiencies. Clin Immunol. 2004;112:1-7.

36. Rojavin MA, Hubsch A, Lawo JP. Quantitative evidence of wearoff effect at the end of the intravenous IgG (IVIG) dosing cycle in primary immunodeficiency. J Clin Immunol. 2016;36:210-9.

37. Hadden RD, Marreno F. Switch from intravenous to subcutaneous immunoglobulin in CIDP and MMN: improved tolerability and patient satisfaction. Ther Adv Neurol Disord. 2015;8:14-9. 